\title{
AN INEQUALITY FOR BOUNDED FUNCTIONS
}

\section{OMRAN KOUBA}

Abstract. In this note we prove optimal inequalities for bounded functions in terms of their deviation from their mean. These results extend and generalize some known inequalities due to Thong (2011) and Perfetti (2011).

Mathematics subject classification (2010): 26B20, 26D25.

Keywords and phrases: Bounded functions, convex functions, inequalities.

\section{REFERENCES}

[1] D. J. H. Garling, Inequalities, A Journey into Linear Analysis, Cambridge University Press, 2007.

[2] O. KoubA, Solution To Problem 23, MathProblems 2, 1 (2012), 61-63.

Online: http://www . mathproblems-ks.com.

[3] P. Perfetti, Proposed Problem 23, MathProblems, 1, 4 (2011), 32-47.

Online: http://www . mathproblems-ks.com.

[4] D. V. Thong, Problem 11581, American Mathematical Monthly 118, 6 (2011), pp. 557. doi:10.4169/amer.math.monthly.118.06.557. 